\title{
A kirigami approach to engineering elasticity in nanocomposites through patterned defects
}

\author{
Terry C. Shyu ${ }^{1,2}$, Pablo F. Damasceno ${ }^{3}$, Paul M. Dodd ${ }^{4}$, Aaron Lamoureux ${ }^{1}$, Lizhi $\mathbf{X u}^{1,2,4}$, \\ Matthew Shlian ${ }^{5}$, Max Shtein ${ }^{1,4,5 \star}$, Sharon C. Glotzer ${ }^{1,2,3,4 \star}$ and Nicholas A. Kotov ${ }^{1,2,3,4 \star}$
}

\begin{abstract}
Efforts to impart elasticity and multifunctionality in nanocomposites focus mainly on integrating polymeric ${ }^{1,2}$ and nanoscale $e^{3-5}$ components. Yet owing to the stochastic emergence and distribution of strain-concentrating defects and to the stiffening of nanoscale components at high strains, such composites often possess unpredictable strain-property relationships. Here, by taking inspiration from kirigami-the Japanese art of paper cutting-we show that a network of notches ${ }^{6-8}$ made in rigid nanocomposite and other composite sheets by top-down patterning techniques prevents unpredictable local failure and increases the ultimate strain of the sheets from 4 to $370 \%$. We also show that the sheets' tensile behaviour can be accurately predicted through finite-element modelling. Moreover, in marked contrast to other stretchable conductors $^{3-5}$, the electrical conductance of the stretchable kirigami sheets is maintained over the entire strain regime, and we demonstrate their use to tune plasma-discharge phenomena. The unique properties of kirigami nanocomposites as plasma electrodes open up a wide range of novel technological solutions for stretchable electronics and optoelectronic devices, among other application possibilities.
\end{abstract}

Strain engineering has become increasingly important for the next generation of nanocomposites, enabling a combination of mechanical and electrical properties typically unattainable in traditional metals, ceramics and polymers ${ }^{2,5,9}$. There is a great need to further expand the range of accessible strain states and develop new ways to deterministically control relationships between strain and physical properties of materials. Typically, it is desirable to retain functional properties under high strains ${ }^{2,3,5}$, but the relationship between strain and desired function (for example, stress, electrical conductivity, transparency, and so on) for elastic nanocomposites can be hard to predict owing to the complexity of deformation and failure mechanisms. It is known also that stress failure of stiff and brittle materials strongly depends on stochastically distributed nanoscale and microscale defects, which serve as stress concentrators that promote propagation of microcracks, leading to failure ${ }^{10}$. Many natural and engineered nanocomposites ${ }^{7,10-14}$ combine hard and soft components that can arrest the propagation of microcracks, but their ultimate strains do not exceed $5 \%$ at high loading of the stiff components. On the other hand, random stress concentrations can be avoided in highly elastic nanocomposites, and one can reach strains as high as $470 \%$, but at the expense of drastically altering functional properties, such as electrical conductivity ${ }^{9,15}$. Interfacial stiffening of polymer chains at interfaces with nanoscale components ${ }^{16-18}$ severely limits the macroscale elasticity of composites, as well as their ability to relax local strain singularities. Strain-induced restructuring in several interdependent components adds to the complexity of the multiscale deformations in nanocomposites, and severely complicates predictive modelling of their tensile behaviour.

In this work we investigate a method to increase the strain capabilities of conductive materials, borrowing the concept from the art of paper cutting known in different cultures as jianzhi, kirigami and monkiri, or silhouette. We shall primarily use the term kirigami because of the greater emphasis of this Japanese technique on repetitive patterns and their effects on three-dimensional (3D) deformations of paper sheets.

Patterns of notches have recently been used to engineer macroscale structures capable of high strains and exhibited other unusual mechanical properties ${ }^{19-23}$. We propose that similar patterns can be used as a tool for materials engineering ${ }^{24,25}$. Indeed, we find that stiff nanocomposite sheets acquire unusually high extensibility after microscale kirigami patterning, the result of stress delocalization over numerous preset deformation points. We also find that the kirigami approach can pave the way towards predictive deformation mechanics for such complex materials as composites and provide a systematic means to engineer elasticity. Moreover, we find that patterning has only a negligible impact on the electrical conductance of macroscale sheets, which enables the use of kirigami nanocomposites as an electrode to control plasma discharge under strain.

In traditional kirigami, cut patterns are introduced into paper sheets to attain a desirable topology on folding. Using standard top-down techniques such as photolithography, we can extend this technique to the micro- or potentially nanoscale (Fig. 1). This fabrication process offers both scalability and accuracy, providing us with a means to produce similar patterns across multiple length scales. Here the homogeneity of material must be commensurate with the length scale of the kirigami cuts, which is true for many nanocomposites, as exemplified by graphene oxide multilayers made by vacuum-assisted filtration (VAF) or layer-bylayer assembly (LBL) techniques (Fig. 1b ${ }^{26}$.

Although many cut patterns are possible, a simple kirigami pattern consisting of straight lines in a centred rectangular arrangement (Fig. 2 inset) made of tracing paper (Young's modulus, $E=1.2 \mathrm{GPa})$ provides an experimentally convenient model for this study (Methods). This pattern has allowed us to develop a comprehensive description of deformation patterns taking place in the material. The original material without patterning shows a strain of $\sim 4 \%$ before failure; its deformation primarily involves

\footnotetext{
${ }^{1}$ Department of Materials Science and Engineering, University of Michigan, Ann Arbor, Michigan 48109, USA. ${ }^{2}$ Biointerfaces Institute, University of Michigan, Ann Arbor, Michigan 48109, USA. ${ }^{3}$ Applied Physics Program, University of Michigan, Ann Arbor, Michigan 48109, USA. ${ }^{4}$ Department of Chemical Engineering, University of Michigan, Ann Arbor, Michigan 48109, USA. 5 Penny W. Stamps School of Art and Design, University of Michigan, Ann Arbor, Michigan 48109, USA. *e-mail: mshtein@umich.edu; sglotzer@umich.edu; kotov@umich.edu
} 
a
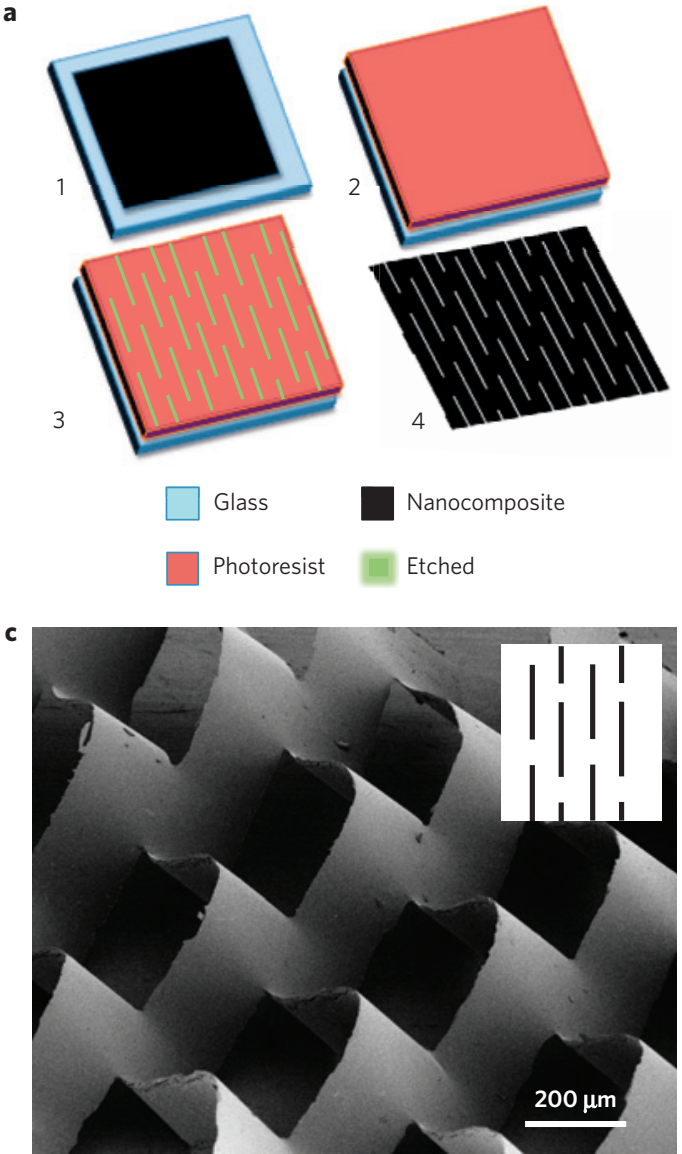

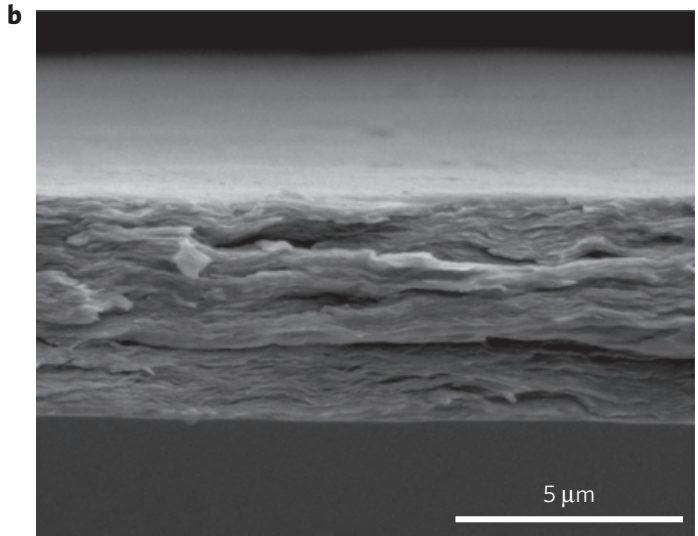

d

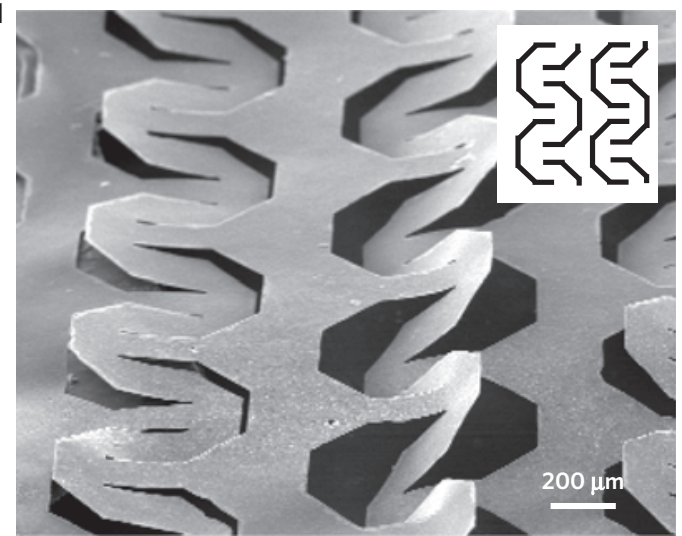

Figure 1 | Microscale kirigami patterns. a, Schematic of the kirigami microfabrication process. (1) A nanocomposite is deposited on a solid substrate for patterning; (2) photoresist is deposited and developed; (3) an oxygen-plasma etch through the nanocomposites creates kirigami patterns; (4) nanocomposite sheet is detached from the substrate. $\mathbf{b}$, Scanning electron microscopy of the cross-section of a GO-PVA nanocomposite. c,d, Two examples of microscale kirigami patterns in GO-PVA nanocomposites after photolithography. The insets show the corresponding kirigami unit cells.

stretching of the individual nano-, micro- and macroscale cellulose fibres within the matrix (Fig. 2 grey curve). With a single cut in the middle of the sample, the stress-strain curve shows a slight decrease of ultimate strength but otherwise behaves similarly to the pristine paper (dashed blue). In contrast, a sheet of the same paper with the tessellated kirigami cuts (green) shows markedly different tensile behaviour. The initial elasticity at $<5 \%$ strain (Fig. 2, purple section) closely follows the deformation curve of the pristine sheet. As the applied tensile force exceeds a critical buckling force, the initially planar sheet starts to deform as the thin struts formed by the cuts open up (Supplementary Fig. 2). Within a secondary elastic plateau regime (Fig. 2, green section), buckling occurs at the struts as they rotate to align with the applied load, and deformation occurs out of the plane of the sample. During the deformation process, kirigamipatterned sheets exhibit out-of-plane deflection due to mechanical bistability (Supplementary Fig. 1) ${ }^{27,28}$. This out-of-plane deflection can be used to impart additional functionality, as we demonstrate in the later part of the present study. Finally, the alignment of the struts causes the overall structure to densify perpendicular to the pulling direction (Fig. 2, white section). Failure then begins when the ends of the cuts tear and crease owing to high stress at these regions.

The effect of the kirigami pattern on the overall mechanical response can be evaluated using beam deflection analysis. We approximate individual struts formed by kirigami as beams (Supplementary Information and Supplementary Fig. 2): the beam length is related to the length of the cut $\left(L_{c}\right)$, the spacing in the transverse direction $(x)$, and the spacing in the axial direction $(y)$. Beam deflection analysis predicts that the critical force scales with $E y t^{3} /\left(L_{c}-x\right)^{3}$, where $E$ is

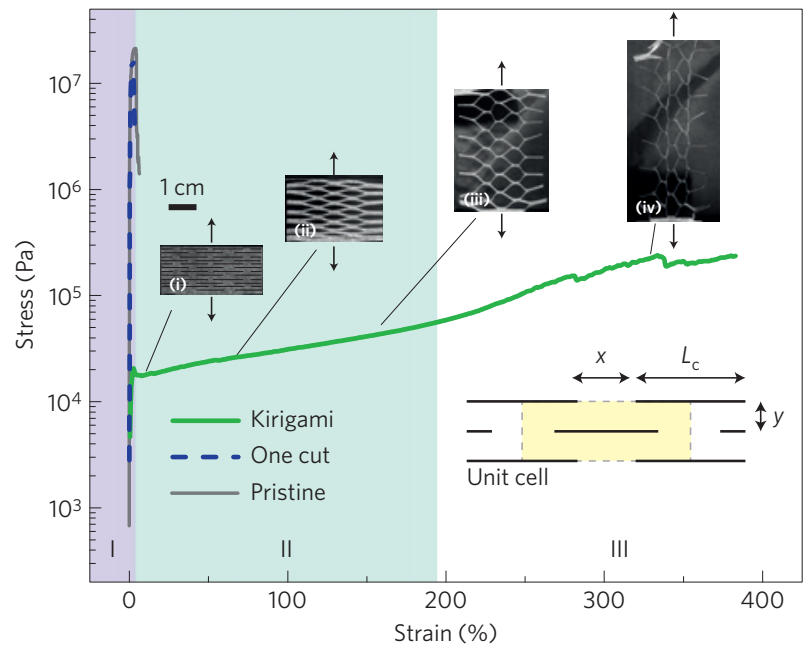

Figure 2 | Stress-strain curves for model macroscale kirigami sheet (green), non-patterned sheet (grey curve), and a sheet with a single notch in the middle (dashed blue). Sections I (purple), II (green) and III (white) represent the regions of initial elastic deformation, secondary elastic deformation with buckling, and pattern collapse, respectively. Scanning electron microscopy images (i-iv) of the deformed kirigami pattern in different regions of the strain-stress curve. Inset: Cut pattern unit cell marked in yellow. Cuts are indicated by the black horizontal lines. The sheets are made on paper using a CAD-programmed computerized cutter. 

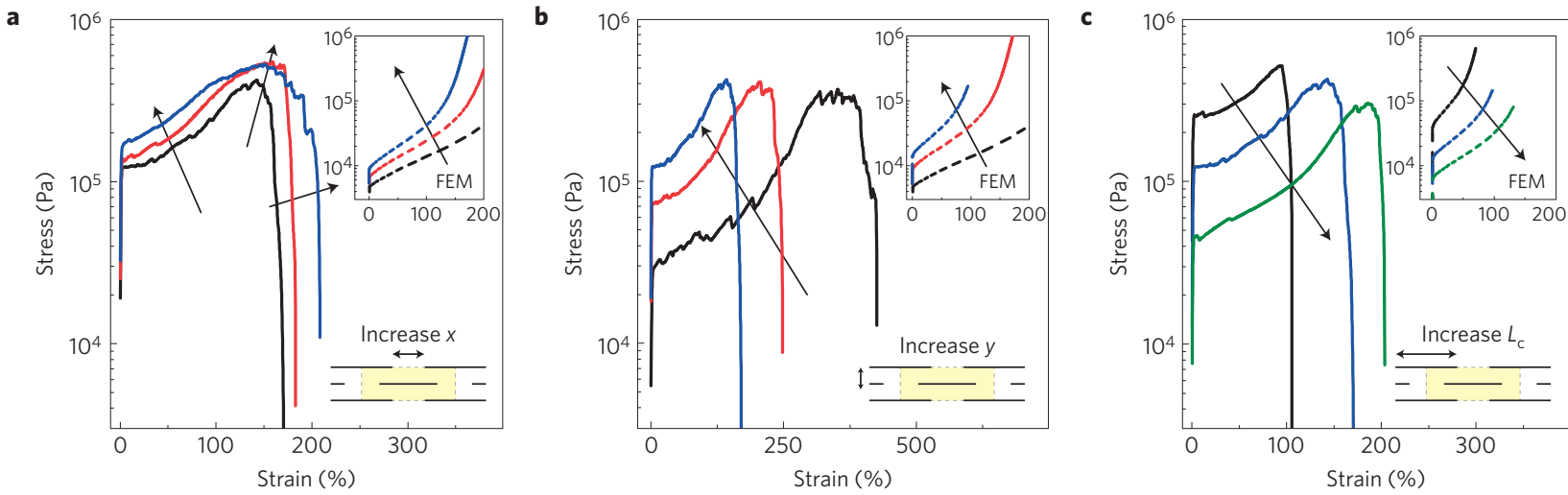

Figure 3 | Experimental and FEM-calculated stress-strain curves for macroscale kirigami sheets with variable unit-cell parameters. a, Horizontal separation $x$ between the cuts. b, Vertical separation $y$ between the cuts. c, Cut length $L_{c}$. Main figures show the experimental results and insets show results from FEM.

Young's modulus, and $t$ is the thickness of the sheet. This approximated force-deflection relationship does not account for deformation in buckling and torsion that is experienced in the sample as a whole, but shows a dependence on the unit-cell geometry. We compare this analysis with experimental results and use finite-element modelling (FEM) to understand the post-buckling behaviour.

Having defined the relevant geometric and material parameters, we investigate the control over the deformation by systematically varying the kirigami unit-cell geometry in the plane view, as defined in Fig. 3a-c (lower insets), for horizontal spacing $x$, vertical spacing $y$, and cut length $L_{c}$, respectively (Supplementary Table I). As expected, the critical buckling load and the size of the nonlinear elastic region-which dictates the maximum extension of the samples at failure-are strongly affected by the unit-cell geometry (Fig. 3a-c). The critical buckling load marks the onset of buckling, where the initial elastic linear regime transitions to the nonlinear regime. Our experimental results show that an increase in $x$-spacing shifts the stress-strain curve up, corresponding to higher critical buckling loads. An increase in $y$-spacing decreases the maximum extension and increases the critical buckling load, as expected from the beam analysis. An increased $L_{\mathrm{c}}$ softens the material, resulting in a lower critical buckling load and higher extensibility. Generally, an increased spacing between the cuts makes the sheet more rigid and imparts a higher critical buckling load, whereas increasing the cut length weakens the material, lowers its critical buckling load, and increases its extensibility. In contrast to the usual trade-off between strength and extensibility, an increased $x$-spacing does not exhibit this trade-off. This is because each cut is able to grow, or tear, along the cut length until neighbouring (in the strain-transverse direction) cuts begin to coalesce, without the overall structure failing. In the process of tearing and final coalescence along the cuts, fracture energy is dissipated while allowing the sheet to extend even further. Hence, the increase of toughness here is related to the distance between the structural features, demonstrating a toughening strategy on a higher length scale. This suggests that the relationship between pattern spacing and mechanical response may be extended to other length scales and materials where high strains are desirable. The uniformity of the material is the only structural limitation for such scaling behaviour that we foresee at present. The cuts need only to be larger than the typical variations in materials composition and properties of the sheet.

The key trends observed in our experiments are replicated by FEM analysis (Fig. 3a-c insets), revealing geometric parameters that are in agreement with our intuitive understanding and the beam deflection analysis. We note that FEM accurately reproduces the general stress-strain response and the experimentally observed effects of geometrical parameters. Conversely, FEM predictions underestimate the buckling load. These deviations are associated with microscale deformations of the material, especially in the apexes of the cuts. The finite-element model does not describe the tearing and breaking that occurs in these areas. Confirming the source of the deviations, we achieved quantitative agreement with our experiments by using a uniform crystalline polyimide film (Supplementary Information), laser-cut to render clean, even widths. These samples were also tested for fatigue life up to 1,000 cycles running to $70 \%$ strain, with an $\sim 18 \%$ strain energy fade (Supplementary Fig. 3). This result shows remarkable damage tolerance and suggests potential reversible and reconfigurable applications for the kirigami patterns.

The FEM results show that the applied load is distributed uniformly throughout the kirigami sheets, rather than concentrating on singularities with random initiation sites (Fig. 4). Thus, high strain is accommodated to improve damage tolerance despite multiple defect sites. This deformation scheme contrasts with the deformation of typical stiff materials, where the presence of any defect acts as a stress concentrator from which cracks propagate and lead to fracture. Considering the deformation in terms of stress fields, we find that kirigami patterns can be used to dictate stress concentrations and effectively control deformation. To further reduce the loads at the cuts, we employ a technique widely used in fracture mechanicsblunting the crack tip using a stress distributing geometry, such as circles (Supplementary Fig. 4). These circular features effectively delay the onset of tearing and lead to a larger operating window for the nonlinear elastic region (Supplementary Fig. 4).

The large strains enabled by the kirigami structures described in this study may have strain-invariant electrical conductance, potentially useful in a variety of devices, including stretchable current collectors and electrodes. As previously mentioned, during buckling deformation the kirigami samples generate an out-ofplane texture that can be used to modulate electronic processes. To make 3D kirigami electrodes, we infiltrate tracing paper with well-dispersed single-walled carbon nanotubes (CNT) or deposit an LBL-assembled (CNT/PVA) $)_{30}$ film on a $5-\mu \mathrm{m}$ parylene (Fig. $5 \mathrm{a}-\mathrm{c}$ and Methods). With the kirigami patterns, the yield strain increases from around $5 \%$ (ref. 29 ) to $\sim 290 \%$ and $\sim 200 \%$ for the paper and LBL nanocomposite, respectively. The conductance does not change significantly during stretching for either the paper or LBL nanocomposites (Fig. 5c), indicating that the presence of the patterned notches accommodates strain while maintaining a conductive network provided by the CNT. In both cases the strain obtained is 20-30 times greater than for similar composites without the notch patterns ${ }^{30}$.

The unique combination of high strain and high conductivity observed for 3D kirigami nanocomposites allows us to use them as 


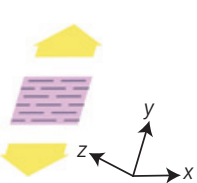

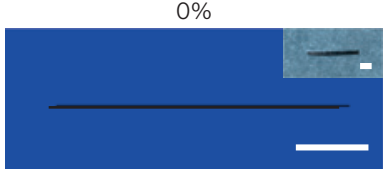

e

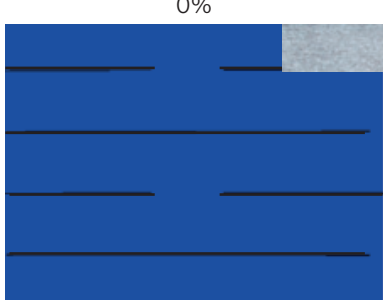

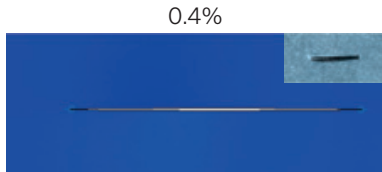

f

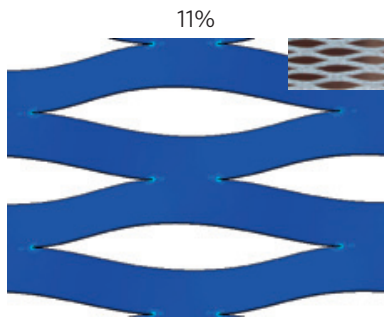

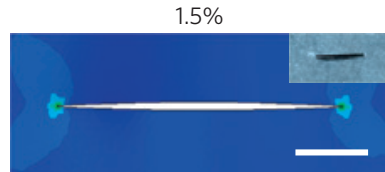

g

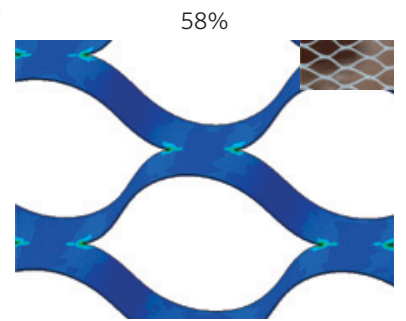

Figure 4 | Stress-concentration visualization in FEM. a, Schematic of loading direction with respect to the unit cell. b-d, FEM snapshots showing stress

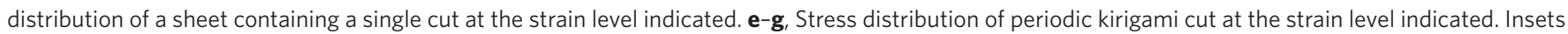
in $\mathbf{b}-\mathbf{g}$ show the corresponding samples. Scale bar, $2 \mathrm{~mm}$.
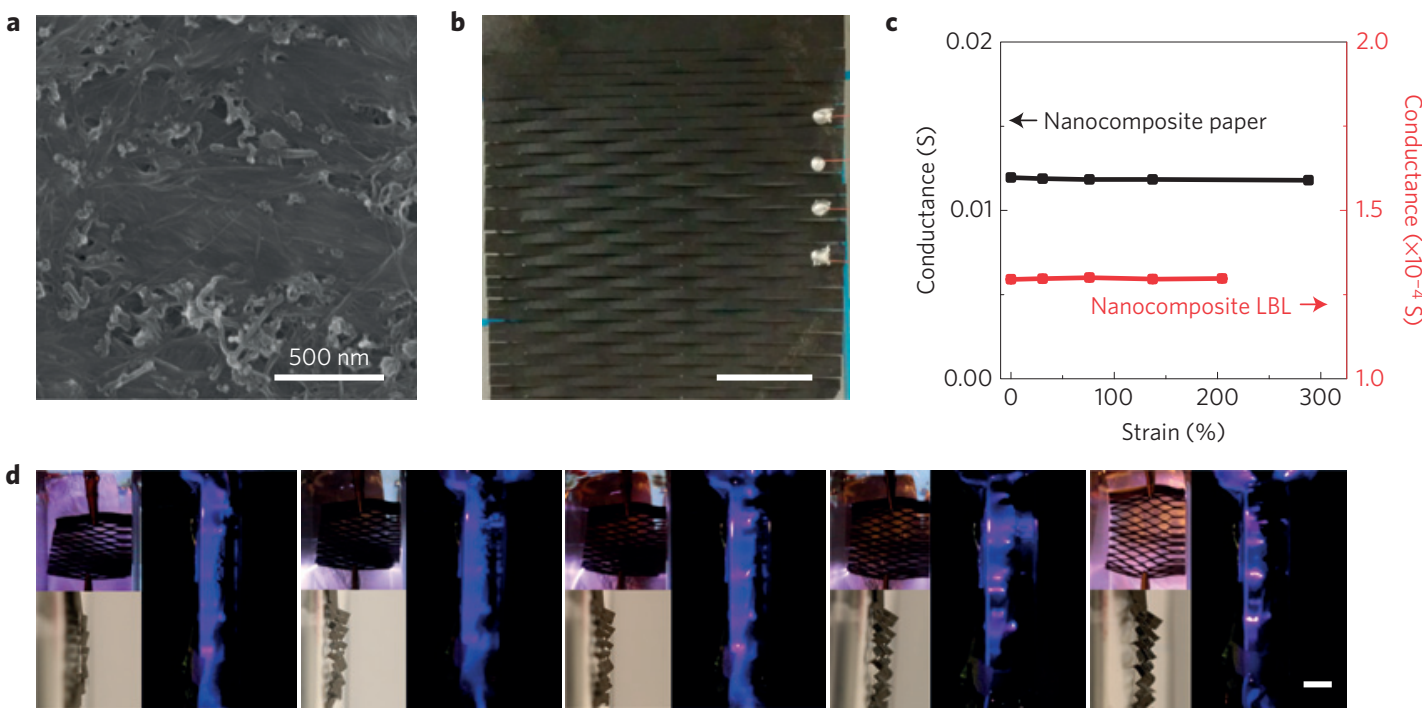

Figure $\mathbf{5}$ | Conducting kirigami nanocomposites. a, Scanning electron micrograph of the CNT nanocomposite sheet made by infiltrating paper with CNT to yield a uniform conducting sheet. b, Nanocomposite sheet following kirigami patterning. c, Conductance versus strain of CNT nanocomposite paper and LBL CNT nanocomposite on parylene. d, Plasma patterns generated on a kirigami electrode at strain values of 5, 10, 15, 20 and 25\% (from left to right); upper and lower insets show front and side views, respectively. Scale bars in $\mathbf{b}$ and $\mathbf{d}$ are $1 \mathrm{~cm}$.

electrodes for tunable plasma discharge inside an argon-filled glass tube. At constant voltage and pressure, the electric field concentrates at the sharp apices that arise from the strain-induced out-of-plane deformation (Fig. 5d). Effectively, the increased strain increases the roughness of the electrode, which lowers the corona onset voltage (Supplementary Information). Hence, as the strain level is increased, we visually observe a higher degree of local ionization and plasma intensity resulting from increased recombination of ionized species. The development of tunable electrodes opens up the possibility of many useful new applications.

In contrast to molecular or nanoscale manipulation of strain, we show that it is possible to control deformation in materials with topdown kirigami patterning, which can be extended to multiple length scales. The new insights obtained here may bridge the gap between nanoscale and macroscale strain engineering, as well as enable novel engineering applications in which out-of-plane deflection can be controlled to create multiscale, reconfigurable structures. Kirigami nanocomposites may find significant use in radio-frequency plasma applications, including surface treatment, materials processing, displays, radar phase arrays, ozone production and corona-induced airflow. Deformable electrodes that can withstand a wide range of strains can also be extended to a variety of flexible-electronic technologies beyond plasma processes.

\section{Methods}

Methods and any associated references are available in the online version of the paper.

Received 20 March 2015; accepted 8 May 2015;

published online 22 June 2015

\section{References}

1. Khang, D-Y. et al. Molecular scale buckling mechanics in individual aligned single-wall carbon nanotubes on elastomeric substrates. Nano Lett. 8, 124-130 (2008).

2. Sekitani, T. et al. A rubberlike stretchable active matrix using elastic conductors. Science 321, 1468-1472 (2008).

3. Zhang, Y. et al. Polymer-embedded carbon nanotube ribbons for stretchable conductors. Adv. Mater. 22, 3027-3031 (2010).

4. Chun, K-Y. et al. Highly conductive, printable and stretchable composite films of carbon nanotubes and silver. Nature Nanotech. 5, 853-857 (2010). 
5. Kim, Y. et al. Stretchable nanoparticle conductors with self-organized conductive pathways. Nature 500, 59-64 (2013).

6. Fratzl, P. \& Weinkamer, R. Nature's hierarchical materials. Prog. Mater. Sci. 52, 1263-1334 (2007).

7. Cranford, S. W., Tarakanova, A., Pugno, N. M. \& Buehler, M. J. Nonlinear material behaviour of spider silk yields robust webs. Nature 482, 72-76 (2012).

8. Meyers, M., McKittrick, J. \& Chen, P. Structural biological materials: Critical mechanics-materials connections. Science 339, 773-779 (2013).

9. Lee, P. et al. Highly stretchable or transparent conductor fabrication by a hierarchical multiscale hybrid nanocomposite. Adv. Funct. Mater. 24, 5671-5678 (2014).

10. Khayer Dastjerdi, A., Rabiei, R. \& Barthelat, F. The weak interfaces within tough natural composites: Experiments on three types of nacre. J. Mech. Behav. Biomed. Mater. 19, 50-60 (2013).

11. Tang, Z., Kotov, N. A., Magonov, S. \& Ozturk, B. Nanostructured artificial nacre. Nature Mater. 2, 413-418 (2003).

12. Barthelat, F. \& Rabiei, R. Toughness amplification in natural composites. J. Mech. Phys. Solids 59, 829-840 (2011).

13. Bauer, J., Hengsbach, S., Tesari, I., Schwaiger, R. \& Kraft, O. High-strength cellular ceramic composites with 3D microarchitecture. Proc. Natl Acad. Sci. USA 111, 2453-2458 (2014).

14. Mirkhalaf, M., Dastjerdi, A. K. \& Barthelat, F. Overcoming the brittleness of glass through bio-inspiration and micro-architecture. Nature Commun. 5, 3166 (2014).

15. Kim, J. Y. \& Kotov, N. A. Charge transport dilemma of solution-processed nanomaterials. Chem. Mater. 26, 134-152 (2014).

16. Jancar, J. et al. Current issues in research on structure-property relationships in polymer nanocomposites. Polymer 51, 3321-3343 (2010).

17. Liff, S. M., Kumar, N. \& McKinley, G. H. High-performance elastomeric nanocomposites via solvent-exchange processing. Nature Mater. 6, 76-83 (2007).

18. Vaia, R. A. \& Wagner, H. D. Framework for nanocomposites. Mater. Today 7, 32-37 (November, 2004).

19. Blees, M., Rose, P., Barnard, A., Roberts, S. \& McEuen, P. L. Graphene Kirigami (2014); http://meetings.aps.org/link/BAPS.2014.MAR.L30.11

20. Castle, T. et al. Making the cut: Lattice kirigami rules. Phys. Rev. Lett. 113, 245502 (2014).

21. Rossiter, J. \& Sareh, S. in Proc. SPIE Vol. 9055 (ed. Lakhtakia, A.) 90550G (Bioinspiration, Biomimetics, and Bioreplication, 2014).

22. Qi, Z., Park, H. S. \& Campbell, D. K. Highly deformable graphene kirigami. Preprint at http://arxiv.org/abs/1407.8113 (2014).

23. Silverberg, J. L. et al. Origami structures with a critical transition to bistability arising from hidden degrees of freedom. Nature Mater. 14, 389-393 (2015).

24. Mullin, T., Deschanel, S., Bertoldi, K. \& Boyce, M. Pattern transformation triggered by deformation. Phys. Rev. Lett. 99, 084301 (2007).
25. Zhang, Y. et al. One-step nanoscale assembly of complex structures via harnessing of an elastic instability. Nano Lett. 8, 1192-1196 (2008).

26. Zhu, J., Zhang, H. \& Kotov, N. A. Thermodynamic and structural insights into nanocomposites engineering by comparing two materials assembly techniques for graphene. ACS Nano 7, 4818-4829 (2013).

27. Holmes, D. P. \& Crosby, A. J. Snapping surfaces. Adv. Mater. 19, 3589-3593 (2007)

28. Kang, S. H. et al. Buckling-induced reversible symmetry breaking and amplification of chirality using supported cellular structures. Adv. Mater. 25, 3380-3385 (2013)

29. Paiva, M. C. et al. Mechanical and morphological characterization of polymer-carbon nanocomposites from functionalized carbon nanotubes. Carbon 42, 2849-2854 (2004).

30. Shim, B. S. et al. Multiparameter structural optimization of single-walled carbon nanotube stiffness, and toughness. ACS Nano 3, 1711-1722 (2009).

\section{Acknowledgements}

This project was sponsored by NSF Grant \#1240264 EFRI-ODISSEI, a joint grant to all senior authors of this work. Parts of the research were also supported by the National Science Foundation, Division of Materials Research Award \# DMR 1120923, and by a Simons Investigator award from the Simons Foundation to S.C.G. The authors gratefully acknowledge fruitful discussions with J. R. Barber on the analytical solution of the buckling kirigami systems. T.C.S. thanks S. R. Spurgeon for stimulating discussions, and R. Hower and H. Zhang for assistance in microfabrication. We thank H. Eberhart for his custom glass apparatus and vacuum system. This work was conducted in part in E. M. Arruda's laboratory, the Electron Microbeam Analysis Laboratory, and the Lurie Nanofabrication Facility at the University of Michigan.

\section{Author contributions}

T.C.S. carried out the experimental set-up, fabrication and measurements. P.F.D. and P.M.D. performed the finite-element modelling. A.L. performed the beam analysis, laser cutting, and the mechanical cycling experiments on Kapton structures. L.X. carried out the LBL assembly. M.Shlian contributed to fabrication and iteration of designs. M.Shtein, S.C.G., and N.A.K. supervised the work. T.C.S., P.F.D. and N.A.K. originated the study, prepared the manuscript, and all authors contributed to data interpretation, discussions and writing.

\section{Additional information}

Supplementary information is available in the online version of the paper. Reprints and permissions information is available online at www.nature.com/reprints.

Correspondence and requests for materials should be addressed to M.S., S.C.G. or N.A.K.

\section{Competing financial interests}

The authors declare no competing financial interests. 


\section{Methods}

Patterning of tracing paper. Tracing paper (Dick Blick Art Materials) was used as obtained. The cutting patterns were first drawn using AutoCAD software, and patterned by a commercial plotter cutter (Graphtec FC4000) onto the paper sheets. The resulting patterned sheets were cut out with scissors for tensile testing.

Synthesis and patterning of nanocomposites. The composite films used for kirigami patterning are graphene oxide (GO)/PVA (Sigma Aldrich, 99\% hydrolysed). Thick composite films (1:2 by dry weight, $E=5 \mathrm{GPa}$ ) were made by vacuum-assisted filtration (VAF), removed from the filter paper and attached to clean glass slide to provide a flat surface for photolithography. Films made in this manner were around 5-6 $\mu \mathrm{m}$ in thickness. For composite films $<1 \mu \mathrm{m}$ thick, layer-by-layer assembly was employed to precisely control the thickness.

The conductive CNT composite films are made with P2-SWNT (Carbon Solutions) of concentration $0.5 \mathrm{mg} \mathrm{ml}^{-1}$, mixed in $2 \mathrm{mg} \mathrm{ml}^{-1}$ poly(sodium styrenesulfonate) (PSS, $\left.M_{\mathrm{w}}=1,000,000\right)$ in deionized water and bath sonicated for $12 \mathrm{~h}$. PSS-modified SWNT solution was poured onto tracing paper and vacuum filtered to infiltrate the SWNT. Kirigami patterns were introduced on the SWNT composite paper. The same kirigami geometry was introduced on a LBL-assembled 30-bilayer nanocomposite made of the same SWNT and polyvinyl alcohol (PVA, $80 \%$ hydrolysed, $\left.M_{\mathrm{w}}=9,000-10,000\right)$ on a glass slide (Supplementary Information). A $5-\mu \mathrm{m}$ parylene layer is vapour deposited onto the 100-nm LBL layer to give a thickness that allows for manual manipulation, and the kirigami pattern is introduced by photolithography.

The photoresist (Megaposit SPR220, Shipley Company) was spin-coated directly onto the film. The patterns were defined by photolithography with a photomask, followed by oxygen plasma etching. The residual photoresist was washed off with acetone, and the free-standing film was removed from the glass slide by soaking in $0.1 \%$ hydrofluoric acid and rinsed with water.
Mechanical testing. Uniaxial tensile tests were performed by means of an RSA 3 dynamic mechanical analyser (DMA) (TA instruments) with a $3.5 \mathrm{~N}$ load cell at a constant strain rate of $0.1 \mathrm{~mm} \mathrm{~s}^{-1}$. The stress-strain data were obtained and each curve was averaged over seven samples.

Finite-element modelling. Commercial finite-element code (Abaqus 6.12) was used to explore a range of geometries in kirigami, which yields insight into the basic mechanisms governing deformation behaviour. The C3D15 element was used with an approximate global mesh size of $0.5 \mathrm{~mm}$. The mesh size is decreased until there is no dependence of the results on the discretization (mesh) size. The standard static procedure was used with a maximum time step of $1 \times 10^{-3} \mathrm{~s}$. We enforce a boundary condition on either end of the kirigami structure: at one end we apply a load in the axial direction and constrain the boundary not to move in either of the orthogonal directions; at the opposite end we place clamped boundary conditions such that no displacement is allowed at the end. To prevent bifurcation of the local buckling, we break the analysis into two steps. In the first step, we apply a small bias force on the top edge of each cut, approximately $1 \times 10^{-5}$ times smaller than the load applied in the axial direction, for the first $5 \%$ of the time steps of the computation. This biases all of the rows to buckle in the same direction but it is small enough not to affect the stress-strain relationship. In the second step of the simulation we pull solely in the axial direction.

Conductance measurements and kirigami electrode. The conductivity as a function of strain was measured using a custom four-probe set-up with copper wires bonded using silver epoxy paint (MG Chemicals). A transformer outputs a voltage of $10 \mathrm{kV}$ at $20 \mathrm{kHz}$ on the sample placed under vacuum at room temperature, and argon (EGL) is introduced until a plasma is formed with a visible purple glow. 